\title{
Energy Dissipation Model for 4G and WLAN Networks in Smart Phones
}

\author{
Shalini Prasad \\ Research Scholar, Jain University \\ Dept. of Electronics and Communication Engineering \\ City Engineering College, Bangalore-560061, India
}

\author{
S. Balaji \\ Center for Engineering Technologies \\ Jain Global Campus, Jain University \\ Jakkasandra Post, Kanakapura Taluk \\ Ramanagara Dist.-562112, Bangalore, India
}

\begin{abstract}
With the modernization of the telecommunication standards, there has been considerable evolution of various technologies to assist cost effective communication. In this regard, the fourth generation communication services or commonly known as 4G mobile networks have penetrated almost every part of the world to offer faster and seamless data connectivity. However, such services come at the cost of energy drained from the smart phone supporting 4G services. This paper presents an algorithm that is capable of evaluating the actual amount of energy being dissipated while using next generation mobile networks. The study also performs a comparative analysis of energy dissipation of $4 \mathrm{G}$ networks with other wireless local area networks to understand the networks that cause more energy dissipation.
\end{abstract}

Keywords-G Wireless Networks; Energy Consumption; Smart phone; Wi-Fi

\section{INTRODUCTION}

There has been a remarkable improvement in the cellular technologies right from handheld devices to services in last 5 years. This phenomenon has lead to a new era of mobile computing that has potential supportability of ubiquitous and pervasive computing. At present, the mobile applications are mushrooming in faster pace in the commercial markets and its adoptability seems to be exponentially higher. The users make use of these mobile applications for multiple purposes: i) entertainment, ii) social networking, iii) business utilities, iv) remote monitoring system, v) educational purpose, vi) public service utilities and many more. Although the usage of such mobile apps makes the work easier and saves lots of productive time, it is done at the cost of battery. Normally, mobile apps with extensive threads use the hardware resources (brightness, contrast, sound, touch etc.) leading to high energy dissipation [1]. With advancement in hardware circuitry and mobile operating system, various features have been evolved that are in compliance with Moore's Law [2] [3] as witnessed in the existing evolution of different processors and circuit design in the existing smart phones. But with the size of the hardware circuits scaling down, the energy dissipation is quite difficult to control and obtaining maximal performance is another challenging factor. There are two prospects in this: (i) novel hardware design to support execution of new mobile applications and (ii) unique networking protocols that is anticipated to support seamless application. The existing wireless standards e.g. IEEE 802.11, 802.16, 802.15, etc. are used for Wireless Local Area networks, 3G, 4G networks respectively. This IEEE standard is now extensively used in creating a network of mobile communications. It should be known that all these IEEE standards do have distinct protocol stacks and unique networking characteristics. Interestingly, the usage of IEEE standard is not uniform in smart phone devices. The prime reason behind this is that smart phone has multiple forms of antenna e.g. main antenna, WLAN antenna, GPS antenna, FM antenna, and diversity antenna. All these antennas use different IEEE standards and specifications that cause excessive energy dissipation. Hence, if the network has more supportability of multicarrier signals e.g. $3 \mathrm{G}$ and $4 \mathrm{G}$ networks than it is quite evident that energy dissipation from battery would be increased. However, the users also switch option between the usage of $3 \mathrm{G} / 4 \mathrm{G}$ network as well as IEEE 802.11 networks for obtaining faster access to Internet-based resources. Usage of IEEE 802.11 standard for wireless access by the cell phone causes faster access even for heavier applications which cannot be seen much in 3G/4G network. A simple example is Skype calls whose quality is quite poor in $3 \mathrm{G}$ and $4 \mathrm{G}$ networks while it is superior in Wi-Fi networks. However, there is an unsolved question i.e. which is energy efficient network 3G/4G or IEEE 802.11 standard. The researcher in [4] has shown that usage of any wireless network works by task sharing and cooperation process in order to balance the load and energy. Therefore, multiple network participation permits decreasing the energy utilization of cell phones and allow the use of a few services, like file streaming or downloading, in addition to web scanning. Additionally, the author in [5] depicts another efficient, method for saving energy in portable VoIP. The presented technique has used GSM system in order to solve the energy consumption problem in WLAN network assisting in making voice calls. This paper discusses about a simple technique based on radio resource control to evaluate energy dissipation in wireless networks. It also provides a few results to demonstrate the effect of $4 \mathrm{G}$ and WLAN networks on the energy utilization of smart phones concentrating on voice services and data association. Further, this paper exhibits a cost efficient approach for dependable measurements of energy on smart phones and provides a modeling technique for scaling the battery consumption over IEEE 802.11 networks or in $4 \mathrm{G}$ networks. Section II briefly explains the background and motivation of the proposed work. It includes cellular power management, Wi-Fi power management, and the measuring methodologies for $4 \mathrm{G}$ and $\mathrm{Wi}-\mathrm{Fi}$, motivation limitations of mobile devices and finally quality of experience perception factors. Section III reviews the 
work being carried out by other researchers. Section IV explains design and implementation of energy consumption model along with an algorithm for the proposed model. Section V presents results and discusses the proposed scheme. Section VI gives concluding remarks and direction for future work is given in Section VII.

\section{BACKGROUND AND MOTIVATION}

Different vendors differentiate among the mobile devices by the services they offer and utilize completely accessible computational energy to offer new services. As shown in Figure 1, as more and more applications and services a smart phones offer, more the energy utilization will be. A phone, when runs out of battery, cannot provide access to mobile services for its users, thus reducing the revenue generated by the service to the service provider. This leads the manufacturers to focus on developing ways to extend battery life and hence the device operational time.

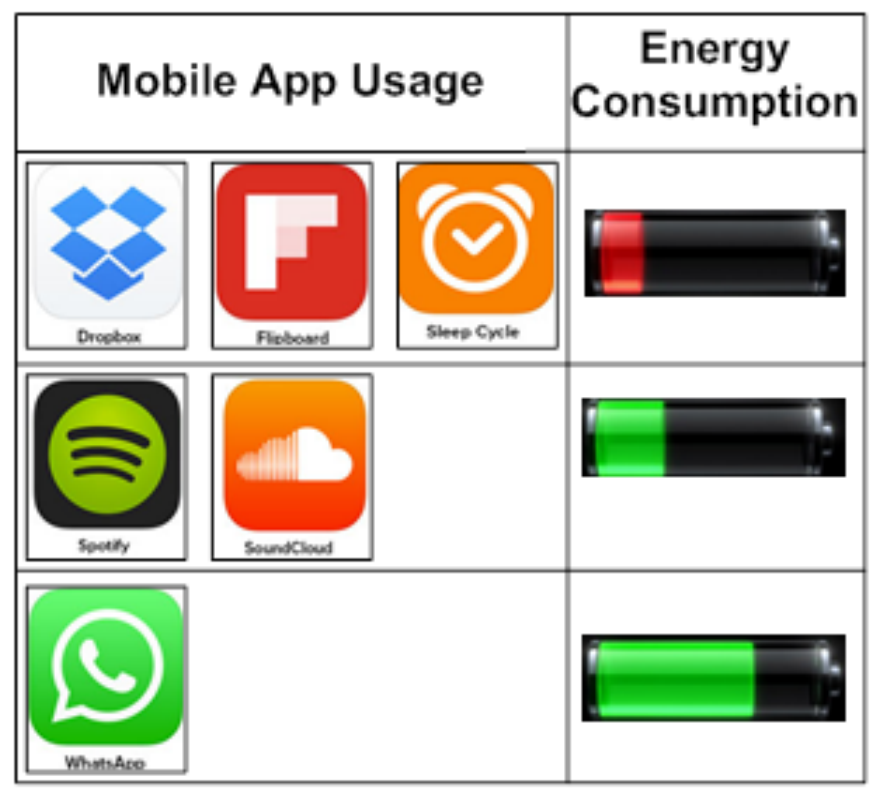

Fig. 1. Energy Consumption by Mobile Applications

\section{A. Background}

In order to retain superior service quality, the existing manufacturers have started emphasizing on energy optimization in smart phones along with associated constraints of size and heat dissipation [6][7]. Basically, every smart phone has three units: processing unit, power unit, and radio (or communication) unit. All these units have varying energy requirements. The energy drainage in the smart phone is controlled by two significant parameters: i) radio resource control and ii) transmission energy. The radio resource control is responsible for managing the control plane and also causes establishment of connection, broadcasting, notification of paging etc. Similarly, the transmission energy is responsible for allocation of energy required for forwarding definite bits of data in a defined communication channel. Both the parameters are dominantly used as the first preference to perform energy management in low power communication devices. Figure 2 shows a description of the radio resource control and its associated mechanism when implemented on existing GSM networks as well as on any WCDMA networks with 4G compliance [5]. The mechanism turns the radio in idle phase when there is no significant activity in a network. This state of idleness also consumes some amount of power. The radio immediately switches to state of high power if an active network is sensed. This principle either uses Forward Access Channel $(\mathrm{FACH})$ or Dedicated Channel $(\mathrm{DCH})$. The prime responsibility of $\mathrm{DCH}$ is to retain the dedicated channel in order to obtain maximized throughput as well as minimized latency. However, all these are achieved at the cost of energy. On the other hand $\mathrm{FACH}$ state is responsible for channel sharing with all available devices. It is preferred option of energy management when there is less availability of traffic for performing transmission. Power consumption capability of $\mathrm{FACH}$ is better in comparison to $\mathrm{DCH}$.

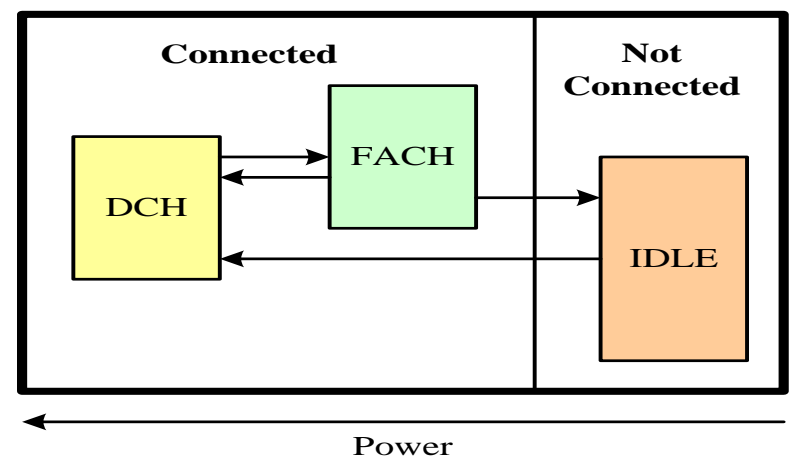

Fig. 2. Radio Resource Control Mechanism

The routers or the access points in the Wi-Fi networks are responsible for controlling the energy dissipation. However, it all depends on i) what type of Wi-Fi network it is? ii) How big it is?, and iii) what protocol is used in routing? An older version of $\mathrm{Wi}-\mathrm{Fi}$ uses IEEE $802.11 \mathrm{a} / \mathrm{b} / \mathrm{g}$ family quite frequently. However, now we have IEEE 802.11 n family too and much higher versions are also available. Majority of them use power up to 6 watts in 24 Hours. The WLAN router does not have many options to save its energy consumption other than turning it off. However, Wi-Fi features available on smart phones use power saving mode to minimize unnecessary energy depletion. According to the concept of power save mode, the size of the data to be transferred and the transmitted power value are directly proportional to amount of energy being considered for data transmission in Wi-Fi networks. The next part of the background discussion is on cellular networks that support $4 \mathrm{G}$ services. The usage of $4 \mathrm{G}$ services make use of LTE (Long Term Evolution) which already provides multiple tools to conserve energy during radio access mechanism in 4G networks. There is also availability of various routing mechanisms that have significant energy conservation properties in $4 \mathrm{G}$.

\section{B. Motivation}

4G telephones offer good services compared with $3 \mathrm{G}$ telephones, particularly with regards to bit rate while downloading or transferring information. Besides, they can support information and voice activity in the meantime permitting video calls, for instance. However, utilization of 
data services is just gradually turning out to be more widespread. Numerous customers still utilize their telephone primarily for voice and Short Message Service (SMS) and very little for data broadband services. Also, numerous areas still have constrained $4 \mathrm{G}$ scope and the telephone persistently makes hand-offs from $3 \mathrm{G}$ to $4 \mathrm{G}$ systems and the other way around as the cell telephones move into and out of $4 \mathrm{G}$ scope. Due to this switch over from $4 \mathrm{G}$ to $3 \mathrm{G}$ and vice versa particularly when no information transmission is required, has a high cost as far as energy utilization is concerned.

The need for Wi-Fi based phones is increasing rapidly due to the ubiquitous presence of WLANs. Power consumption is the vital issue in the selection of a mobile phone [9]. Since all these devices make use of rechargeable batteries, the attractive services and features unfortunately drain lot of energy stored in a capacity limited battery of a smart phone. Achieving low power consumption for wireless devices has become a key design issue [10] which motivated us to carry out this research. For any mobile device, the major constraints are battery life, size and weight [11]. Such handheld devices will require enough power to perform processing. However, they attempt to minimize energy consumption during idle networking conditions. Discussion on energy saving schemes on such devices can be seen in [12] [13]. In [14], recommendations for amplifying the battery life of an Android device are given. It is a typical issue among smart phone manufactures in figuring out ways to expand battery life of their device and permit clients to utilize portable administrations for a more drawn out time [12]. This is also another motivation behind carrying out this research. Though mobile devices are making it easier for users to communicate, many users get frustrated while accessing certain applications and services using their mobile devices. The technological advancements of mobile device are new and yet they are improving [15]. Certain limitations of the mobile devices are:

\section{a) Limited memory \\ b) Limited processing power \\ c) Battery consumption \\ d) Simplicity \\ e) Accessibility}

Apart from technical specifications, it is also essential that various factors should be used to scale the experience of user. This perception is termed as QoE which determines the usability of the service or application in subscriber's perspective. The following are some of the critical perception factors of QoE [16]
a) Speed
b) Accessibility
c) Session quality
d) Integrity
e) Flexibility

The way clients perceive the execution of a system or a mobile service is an ultimate method for measuring that specific administration or system. This perception is named as QoE and is a definitive method for measuring that specific administration or system. The next section discusses about the existing techniques that have emphasized on the various techniques for conserving energy with respect to various network-based services.

\section{RELATED WORK}

A few studies have proposed models for assessing the energy utilization of mobile services. In any case, as far as we are concerned, proposed model is the first outline stage energy utilization estimation model considering the different energy utilization properties like signalling and media exchanges. The related work depicted below clearly explains the advance energy consumption schemes used in the past.

Gross et al. [17] present a study that can compute the cumulative energy being drained in a smart phone which was done on the basis of component-based modeling. The study has shown the capability of the device to do so by approximately $4.7 \%$ of error. Another study by Haverinen et al. [18] showcase an impact of certain forms of messages and notifications on the lifetime of WCDMA networks. Usually such forms of networks are capable of faster data delivery with high throughput. The experiment performed by the author shows radio resource control is highly influenced by the energy being consumed. Vegara et al. [19] present a tool called Energy Box which can measure the energy consumption in the devices connected in $3 \mathrm{G}$ networks or in Wi-Fi Networks. According to the author, the traffic pattern is responsible for the energy being consumed over such mobile networks. The study shows an accuracy of $99 \%$ in energy computation for both the networks.

Balasubramanian et al. [20] present a mechanism to evaluate the energy being consumed in utilizing regular GSM networks as well as Wi-Fi networks. The computation of the energy was carried out with respect to the overhead in tail energy. The significant contribution of the study is to formulate a protocol called Tail Ender for minimizing the energy dissipation in mobile applications. Kelenyi et al. [21] make use of distributed hash tables to analyze the energy being spent by the mobile phones. Lane et al. [22] compute energy in smart phone equipped by sensors. The technique allows aggregation of the sensory data from the cellular phone to minimize the energy overhead for the user. The mechanism allows collection of the active usage data to develop a decisive model for energy conservation. The studies carried out by Damasevicius et al [23] have used the concept of measuring the energy consumed due to running of multiple applications on mobile device. The study also uses 3DMark06 (a benchmarking tool) to measure the effectiveness of the technique. Perala et al. [24] present a tool that can compute the extent of energy utilization on WCDMA networks governed by radio resource control.

Han et al. [25] present a scheme for energy being consumed in smart phones. Study on energy consumption over IEEE $802.11 \mathrm{~g}$ network by Xiao et al. [26] and nearly similar direction of study was also carried out by Zhang et al. [27]. Study towards energy efficiency in $4 \mathrm{G}$ networks as well as a WLAN network was done by Harjula et al. [28]. The author presents a sophisticated model for testifying the impact of protocols residing in application layer on the energy dissipation 
of a cellular phone. Miranda et al. [29] investigate the role of transport layer security on the energy consumption factor of the cellular phones. Similar work was also carried out by Abbas et al. [30]. However, the authors have incorporated a machine learning-based algorithm. The technique also uses crossvalidation mechanism for evaluating the effectiveness of the mechanism. The study carried by Le et al. [31] presents an investigation of multiple radio-technologies used in advanced mobile networks and studied the energy usage over uplink and downlink transmission. Ravi et al. [32] have shown the possible use of cloudlets to minimize massive energy consumption in $3 \mathrm{G}$ and $5 \mathrm{G}$ mobile networks. The technique has investigated the handoff mechanism considering multiple parameters e.g. bit rate, signal strength, and number of interaction. Fuzzy logic was applied to further strengthen the decision. Wang et al. [33] and Sun et al. [34] have performed research in similar lines to minimize the energy consumption due to $4 \mathrm{G}$ and $\mathrm{Wi}-\mathrm{Fi}$ networks.

Thus, it can be seen that there are many researchers working on the existing systems to address the energy dissipation problems while accessing the data from the mobile devices using wireless networks like WLAN, 3G, and 4G. However, a closer look into the existing systems will also show that majority of the techniques have used radio resource control but did not develop a mathematical model to quantize the measurements. Hence, to bridge this gap, we propose a simple mathematical model which is illustrated in the next section.

\section{DESIGN AND IMPLEMENTATION OF ENERGY CONSUMPTION MODEL}

This section discusses about a simple computational model that can perform evaluation of energy on mobile devices due to the usage of mobile networks. This discussion of the proposed system was carried out with respect to two distinct algorithms.

\section{A. Energy Consumption Model}

The core objective of this model is to investigate signalling properties of mobile networks and their possible connections with energy dissipation. We take the case study of standard IEEE 802.11 network and $4 \mathrm{G}$ network protocols as the communication media whose signalling properties will be assessed. The proposed system does not use any form of offline or pre-stored communication data in order to perform computation of energy being dissipated from devices. We develop a computational model with a backbone design of analytical model considering the signalling properties of both the types of the mobile networks. We also consider radio resource control and its associated features with respect to the mobile networks of Standard IEEE 802.11 and 4G networks. The presented technique also considers the inherent characteristics of data transfer of both the mobile networks. . The simulation of the proposed logic was carried out using Matlab where we developed user interface for both client and server. The design principle of the study considers monitoring the energy usage based on the multiple applications running on the mobile devices. The study considers a cut-off based scheme deployed over an interval of the transmission of the data packets. We anticipate that our mechanism will bring better probability of the minimization of an energy being consumed with better linearity on the energy curves. We check for multiple prioritized applications and their respective threads running on the mobile devices. The study was conducted based on end-to-end monitoring of the energy factor on the mobile devices that is connected by wireless communication media of Standard IEEE 802.11 or 4G networks.

The discussion of the technique in the form of algorithm is showcased here that is developed for evaluating the energy on the mobile device.

\section{Algorithm-1}

Input: Fs, Ds, R_Ip, R_Port, Rec_port;

Output: D_matrix, Id_list;

1. Start

2. Initialize all the input parameters;

3. get Pkt_Size;

4. compute $S^{\text {th }}$ value;

5. if $($ Pkt_Size $=$ Th $)$

6. $P k t++$;

7. read Id_list and D_matrix;

8. End;

9. if (Pkt_Size < 0$)$

10. Packet bad size;

11. End;

12. If $\left(P k t \_S i z e<T h\right)$

13. $\mathrm{P}_{\text {tra }}(\mathrm{k})=\mathrm{X} * \mathrm{~S}_{\mathrm{sig}}-\mathrm{Y} * \mathrm{k}+\mathrm{Z}$;

14. End;

15. If $\left(S_{\text {sig }}<S_{t h}\right)$

16. $P_{\text {fach }}(k)=\frac{k_{1}}{k} \cdot\left[P_{\text {tra }}\left(k_{1}\right)-P_{\text {fach }}\right]+\mathrm{P}_{\text {fach }}$,;

17. End;

18. If (Pkt_Size > $=$ Th)

19. $P_{\text {idl }}(k)=\frac{k_{3}}{k} \cdot\left[P_{\text {tra }}\left(k_{3}\right)-P_{\text {idl }}\right]+\mathrm{P}_{\text {idl }}$;

20. End;

21. End;

Algorithm-1 presents a technique based on signalling properties of wireless channels. The overall algorithm is summarized in Algorithm-1 and Algorithm-2. Algorithm-1 gives the algorithm for the upload WLAN system. It uses Fs, Ds, R_Ip, R_Port, Rec_port as inputs and the output is a data matrix and an ID list. Here, the first step is to initialize the input parameters at the server side GUI and select simulation option, WLAN or $4 \mathrm{G}$, and enter the values for remote IP address, remote port number, received port number, average signal frequency, average data size and data transfer rate. If the packet size is equal to the threshold value, then increase the packet size and read the ID list and data matrix. If the packet size is less than zero value, the input is bad packet size. Then discard the packet. If the packet size is less than threshold value, then compute the equation (1) given below:

$$
\mathrm{P}_{\text {tra }}(\mathrm{k})=\mathrm{X} * \mathrm{~S}_{\text {sig }}-\mathrm{Y} * \mathrm{k}+\mathrm{Z} \text {, where } \mathrm{k} \leq \mathrm{k}_{1}
$$

then similarly, if the packet size is less than the signal threshold value, then compute the equation (2) given below:

$$
P_{\text {fach }}(k)=\frac{k_{1}}{k} \cdot\left[P_{\text {tra }}\left(k_{1}\right)-P_{\text {fach }}\right]+\mathrm{P}_{\text {fach }}, \text { where } \mathrm{k}_{1} \leq \mathrm{k} \leq \mathrm{k}_{3}
$$

Finally check if packet size is greater than or equal to threshold value; then compute equation (3) given below:

$$
P_{\text {idl }}(k)=\frac{k_{3}}{k} \cdot\left[P_{\text {tra }}\left(k_{3}\right)-P_{\text {idl }}\right]+\mathrm{P}_{\text {idl }}, \text { where } \mathrm{k} \geqslant \mathrm{k}_{3}
$$


where, $\mathrm{k}, \mathrm{k}_{1}, \mathrm{k}_{2}$ and $\mathrm{k}_{3}$ are the threshold values. In the above equation $\mathrm{P}_{\text {tra }}(\mathrm{k})$ represents transmission power of the signal, the variable $\mathrm{P}_{\text {fach }}(\mathrm{k})$ represents $\mathrm{FACH}$ power consumption of the system, while the variable $\mathrm{P}_{\mathrm{idl}}(\mathrm{k})$ represents ideal power dissipation of the signal. Then, $\mathrm{X}, \mathrm{Y}$ and $\mathrm{Z}$ are the scaling factors.

Algorithm2 shows the server part of the proposed system. Here the data is uploaded through the wireless LAN.

\section{Algorithm-2}

Input: Fs, Ds, R_Ip, R_Port, Rec_Port;

Output: D_matrix, Id_list;

1. Start

2. Initialize all the input parameter;

3. Get Pkt_Siz;

4. Select network;

5. Initialize R_Ip, R_Port_No, Recv_Port_No, Avg_Fs, Avg_Pkt_Size, D_Tr;

6. Get $\mathrm{msg}$;

7. If $(W L A N=1)$

8. Calculate Msg_In;

9. Download_WLAN;

10. End;

11. If $(4 G=1)$

12. Calculate $M s g \_I n$;

13. Download_4G;

14. End;

15. Data downloaded/received successfully;

16. End;

Algorithms-2 gives the steps for server side and client side respectively. In this procedure the input is taken as Fs, Ds, R_Ip, R_Port, Rec_Port, msg, D_flg, Msg_In; at the output side is data matrix and ID list. First initialize all parameters and then start at step 2 and 3 of Algorithm-2. Then select the input packet size along with message input, select WLAN option and calculate the message input value. Then upload the WLAN side. If $4 \mathrm{G}$ is selected directly upload the $4 \mathrm{G}$ message input. Finally, download or receive successfully the uploaded input message.

Table-1 shows the variables used in this algorithm for different input and output side.

TABLE I. NOTATIONS USED

\begin{tabular}{|l|l|l|}
\hline \multicolumn{1}{|c|}{ Sl. No. } & \multicolumn{1}{|c|}{ Variables } & \multicolumn{1}{c|}{ Description } \\
\hline 1. & Fs & Signal frequency \\
\hline 2. & Ds & Data Size \\
\hline 3. & R_Ip & Remote IP \\
\hline 4. & R_port & Remote Port \\
\hline 5. & Rec_port & Received port \\
\hline 6. & D_matrix & Data matrix \\
\hline 7. & Id_list & Id list \\
\hline 8. & Pkt_Size & Packet size \\
\hline
\end{tabular}

\begin{tabular}{|l|l|l|}
\hline 9. & Sth & Strreshold \\
\hline 10. & Th & Threshold \\
\hline 11. & S $_{\text {sig }}$ & S $_{\text {sig }}$ \\
\hline $12 .$. & Avg_Fs & Average Signal Frequency \\
\hline 13. & D_Tr & Data Transfer Rate \\
\hline 14. & Msg_In & Input Message \\
\hline 15. & D_flg & Data flag \\
\hline
\end{tabular}

V. RESULTS AND DisCUSSION

The results we obtained are discussed in this section.

A. Server Side

The inputs given to server side are:

a) Choose: Simulation Option

b) Choose Device Specific: WLAN Network Option

c) Enter Remote IP: local host (you can also enter System IP)

d) Enter the Remote Port No: 300

e) Enter the Received Port No: 301

f) Average Sign Frequency: 200

g) Average Pkt Size (bytes): 250

h) Data Transfer (kbs): 15,000

\section{B. Client Side}

The inputs given to client side are:

a) Choose: Simulation Option

b) Choose Device Specific: WLAN Network Option

c) Enter Remote IP: local host (you can also enter System IP)

d) Enter the Remote Port No: 301

e) Enter the Received Port No: 300

f) Average Sign Frequency: 200

g) Average Pkt Size (bytes): 250

h) Data Transfer (kbs): 15,000

The results analysis is carried out on the basis of an energy being dissipated from the client module. Figure 3 highlights the energy consumption trend for the proposed system with increasing time scale. The assessment of the proposed model is accomplished by transmitting a test data in the distinct wireless channel of standard IEEE 802.11 and $4 \mathrm{G}$ networks. The trend of Figure 3 shows that in idle state power consumption is quite low while in an upload condition the power consumption is found increasing linearly and then it maintains a better linear behavior for 20-80 seconds of time limit. The trend is then found to decrease in its overhead at the same time. The trend eventually shows that power consumption lowers down in the state of network. 


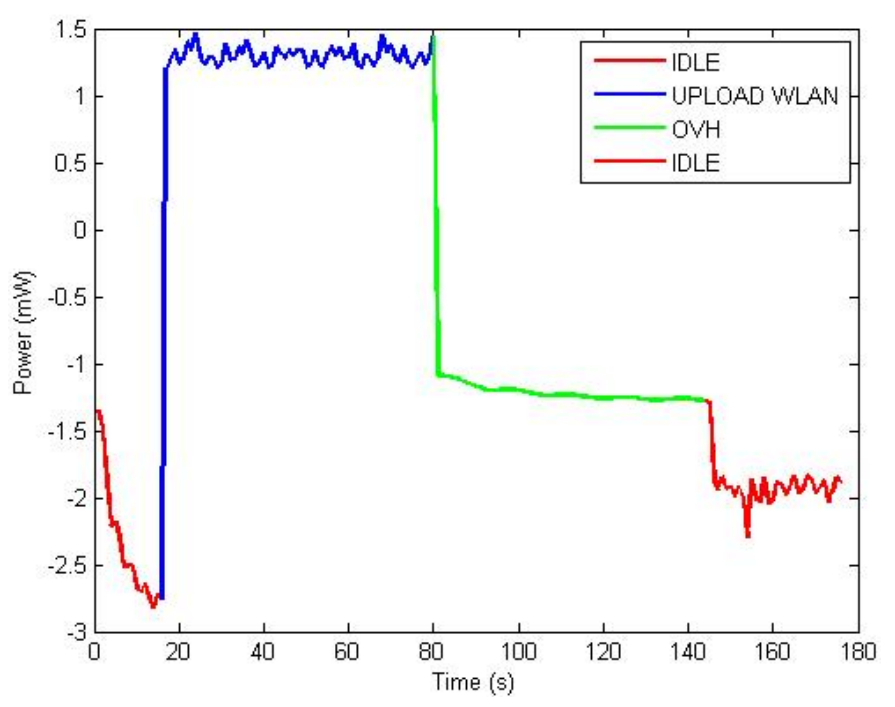

Fig. 3. Power (mW) Vs. Time (s)

We have also performed a comparative analysis of the proposed model in the context of Wi-Fi networks and $4 \mathrm{G}$ networks with respect to lookup time in terms of hours as shown in Figure 4. The study shows that energy dissipation is more for $4 \mathrm{G}$ networks in contrast to Wi-Fi networks. The same test file is forwarded twice in both networks in order to check the rate of energy dissipation effectively. Both uplink as well as downlink transmission were tested for this purpose and it was found that there are frequent switch overs of $\mathrm{FACH}$ to DCH by the $4 \mathrm{G}$ network which has resulted in exponential increase of energy consumption in comparison to Wi-Fi networks.

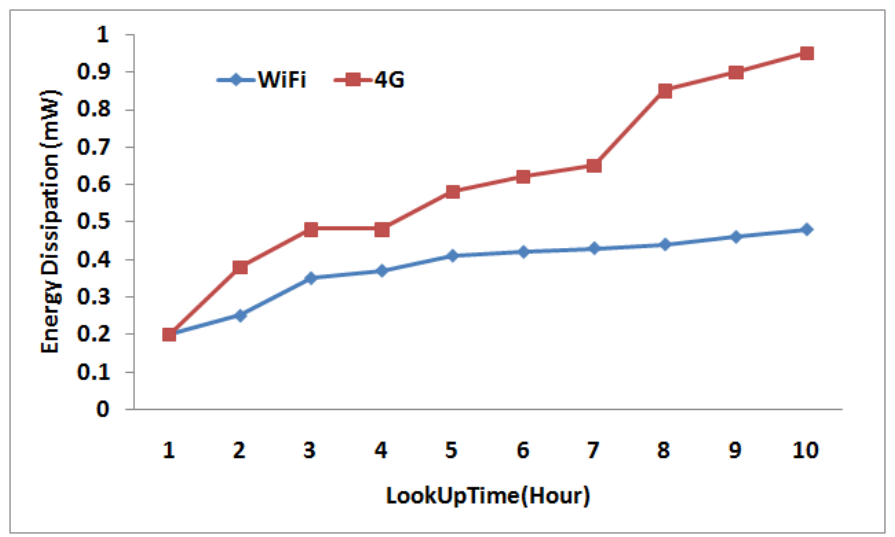

Fig. 4. Comparative Analysis on Different Networks

\section{CONCLUSION}

Energy consumption is one of the critical problems in smart phones and successful execution of multiple mobile applications calls for extensive battery lifetime as well as energy conservation schemes. The biggest problem with the smart phone devices is to understand the importance of various threads running inside the applications. The existing energy conservation schemes call for suppressing some threads leading to temporary minimization of energy which has potential impact on application performance on the mobile device. Therefore, a simple and yet robust framework is required that can study the signaling properties of the usage of mobile networks and then perform investigation on the energy drainage. We argue that a precise detection of energy dissipation will always assist the energy saving schemes. In this research manuscript, we present one such computational model that is capable of measuring accurate energy drainage rate.

\section{FUTURE WORK}

The study towards the future direction will be to develop a model for compensating the energy that was found to be dissipating. As the presented model is capable of assessing cumulative energy drainage owing to wireless local area network and 4G, the study could now trace the priority by identifying the applications or services consuming more energy and choose to suppress those applications for balancing the necessary power. As $4 \mathrm{G}$ services also offer increasing data transfer, we will investigate a better possibility of antenna management techniques for compensating the energy loss.

\section{REFERENCES}

[1] G.P. Perrucci, F. H.P Fitzek, G. Sasso, W. Kellerer, and J. Widmer, "On the impact of $2 \mathrm{G}$ and $4 \mathrm{G}$ network usage for mobile phones' battery life", In Wireless Conference, European, pp. 255-259, 2009

[2] Y. Borodovsky, "Marching to the beat of Moore's Law." In SPIE 31st International Symposium on Advanced Lithography,International Society for Optics and Photonics, pp. 615301-615301, 2006

[3] K.D. Sattler, "Handbook of Nanophysics: Nanomedicine and Nanorobotics", CRC Press, pp. 887, 2010

[4] F. Fitzek and M. Katz, editors. Cooperation in Wireless Networks: Principles and Applications - Real Egoistic Behavior is to Cooperate, ISBN 1-4020-4710-X. Springer, April 2006.

[5] G. Perrucci, F. Fitzek, G. Sasso, and M. Katz. Energy saving strategies for mobile devices using wake-up signals. In MobiMedia - 4th International Mobile Multimedia Communications Conference, Oulu Finland, 2008.

[6] G. P. Perrucci, "Energy Saving Strategies on Mobile Devices," Ph. D. Dissertation, Dept. Multimedia information and Signal Processing, Aalborg University, Denmark, 2009.

[7] Miao, Guowang, Himayat, Nageen, Li Ye, Swami, Ananthram, " Cross Layer OPtimization for Energy-E_cient Mobile Networking: A survey," Wireless Communication and Mobile computing, vol. 9, pp. 529-542, Apr. 2009.

[8] G. Perrucci, F. Fitzek, G. Sasso, and M. Katz. Energy saving strategies for mobile devices using wake-up signals. In MobiMedia - 4th International Mobile Multimedia Communications Conference, Oulu, Finland, 2008.

[9] A. Gupta, P. Mohapatra, "Energy Consumption and Conservation in WiFi Based Phones: A Measurement-Based Study," in 4th Annual IEEE Communications Society Conference on Sensor, Mesh and Ad-Hoc Communications and Networks, 2007, pp.122-131.

[10] Hyun-Ho Choi, Jung-Ryun Lee, Dong-Ho Cho, "On the use of a powersaving mode for mobile VoIP devices and its performance evaluation,"IEEE Transactions on Consumer Electronics, vol. 55, no. 3, pp. 1537-1545, Aug. 2009.

[11] R. Want, "When Cell Phones Become Computers," Pervasive Computing, IEEE, vol. 8, no. 2, pp. 2-5, Apr. 2009.

[12] G. P. Perrucci, F. H. P. Fitzek, G. Sasso, W. Kellerer, J. Widmer, "On the impact of $2 \mathrm{G}$ and $4 \mathrm{G}$ network usage for mobile phones' battery life," Wireless Conference, Europe, pp. 255-259, May 2009.

[13] Battery Life, (Online: Veri_ed January, 2011). Available: http://android.bigresource.com/Samsung/-Vibrant-Battery-indicator-iswrong--hxAgM7rn.html. Accessed on $11^{\text {th }}$ July 2016.

[14] Maximising Battery Life, (Online: Veried December, 2010).Available:http://www.howtogeek.com/howto/25319/complete- 
guide-to-maximizing-your-android-phones-attery-life/. Accessed on $11^{\text {th }}$ July 2016.

[15] Limitations of Mobile Devices, (Online: Veri_ed April, 2011). Available:http://www.wireless-center.net/Mobile-andWireless/2505.html. Accessed on $11^{\text {th }}$ July 2016.

[16] Yu Du, Wenan Zhou, Jian Liu, Junde Song, "A study on the extraction and application of customer perception indexes in mobile network," in IEEE International Symposium on IT in Medicine \& Education, 2009, pp.436-440.

[17] C. Gross, F. Kaup, D. Stingl, D. Richerzhagen, D. Hausheer, and R. Steinmetz, "EnerSim: An energy consumption model for large-scale overlay simulators", IEEE-Local Computer Networks, pp. 252-255, 2013.

[18] H. Haverinen, J. Siren, and P. Eronen, "Energy Consumption of AlwaysOn Applications in WCDMA Networks," in, IEEE Vehicular Technology Conference, Dublin, Ireland, 2007, pp. 964-968.

[19] E.J. Vergara, and S.N. Tehrani, "Energybox: A trace-driven tool for data transmission energy consumption studies," Energy Efficiency in Large Scale Distributed Systems. Springer Berlin Heidelberg, pp. 19-34, 2013.

[20] N. Balasubramanian, A. Balasubramanian, and A. Venkataramani, "Energy Consumption in Mobile Phones: A Measurement Study and Implications for Network Applications", in, ACM Internet Measurement Conference, Chicago, USA, 2009, pp. 280-293.

[21] I. Kelenyi and J.K. Nurminen, "Energy Aspects of Peer Cooperation Measurements with a Mobile DHT System,' in, IEEE International Conference on Communications, Beijing, China, 2008, pp. 164-168.

[22] N. D. Lane, Y. Chon, L. Zhou, Y. Zhang, F. Li, "Piggyback Crowd Sensing (PCS): energy efficient crowd sourcing of mobile sensor data by exploiting smart phone app opportunities", Proceedings of the 11th ACM Conference on Embedded Networked Sensor Systems, 2013.

[23] R. Damasevicius, V. Stuikys, and J. Toldinas, "Methods for measurement of energy consumption in mobile devices", Metrology and Measurement Systems. No. 3, pp.419-430, 2012.

[24] P.H.J. Perälä, A. Barbuzzi, G. Boggia, K. Pentikousis, "Theory and Practice of RRC State Transitions in UMTS Networks," in, IEEE Broadband Wireless Access Workshop, Hawaii, USA, 2009, pp.1-6.

[25] H. Han, J. Yu, H. Zhu, Y. Chen, Y, "Energy-efficient engine for frame rate adaptation on smart phones,". Proceedings of the 11th ACM Conference on Embedded Networked Sensor Systems, Vol.15, 2013.
[26] Y. Xiao, P. Savolainen, A. Karppanen, M. Siekkinen, and A. Ylä-Jääski, "Practical power modeling of data transmission over $802.11 \mathrm{~g}$ for wireless applications" in, International Conference on Energy-Efficient Computing and Networking,Passau, Germany, 2010.

[27] L. Zhang, B. Tiwana, Z. Qian, Z. Wang, R.P. Dick, Z.M. Mao, and L. Yang, "Accurate Online Power Estimation and Automatic Battery Behavior Based Power Model Generation for Smart phones," in, International Conference on Hardware-Software Codesign and System Synthesis, Scottsdale, USA, 2010, pp. 105-114.

[28] Harjula, E.; Kassinen, O.; Ylianttila, M., "Energy consumption model for mobile devices in 4G and WLAN networks," in Consumer Communications and Networking Conference (CCNC), 2012 IEEE, vol., no., pp.532-537, 14-17 Jan. 2012.

[29] Miranda, P.; Siekkinen, M.; Waris, H., "TLS and energy consumption on a mobile device: A measurement study," in Computers and Communications (ISCC), 2011 IEEE Symposium on , vol., no., pp.983989, June 28 2011-July 12011.

[30] Abbas, N.; Taleb, S.; Hajj, H.; Dawy, Z., "A learning-based approach for network selection in WLAN/4G heterogeneous network," in Communications and Information Technology (ICCIT), 2013 Third International Conference on , vol., no., pp.309-313, 19-21 June 2013.

[31] Le Wang; Manner, J., "Energy Consumption Analysis of WLAN, 2G and 4G interfaces," in Green Computing and Communications (GreenCom), 2010 IEEE/ACM Int'l Conference on \& Int'l Conference on Cyber, Physical and Social Computing (CPSCom), vol., no., pp.300307, 18-20 Dec. 2010.

[32] Ravi, A.; Peddoju, S.K., "Mobility managed energy efficient Android mobile devices using cloudlet," in Students' Technology Symposium (TechSym), 2014 IEEE, vol., no., pp.402-407, Feb. 28 2014-March 2 2014.

[33] Yumei Wang; Lin Zhang; Hongyu An; Bin Xu; Geng Xi, "Power consumption testing and optimization for mobile router based on data aggregation and compression," in Wireless Personal Multimedia Communications (WPMC), 2013 16th International Symposium on , vol., no., pp.1-5, 24-27 June 2013.

[34] L. Sun, R.K. Sheshadri, W. Zheng, and D. Koutsonikolas, "Modeling WiFi Active Power/Energy Consumption in Smart phones," IEEE-34th International Conference on Distributed Computing Systems, pp. 41-51, 2014 